\title{
Le Dictateur de Charlie Chaplin ou le rire de la raison
}

\author{
Avertissement \\ Le contenu de ce site relève de la législation française sur la propriété intellectuelle et est la propriété exclusive de \\ l'éditeur. \\ Les œuvres figurant sur ce site peuvent être consultées et reproduites sur un support papier ou numérique sous \\ réserve qu'elles soient strictement réservées à un usage soit personnel, soit scientifique ou pédagogique excluant \\ toute exploitation commerciale. La reproduction devra obligatoirement mentionner l'éditeur, le nom de la revue, \\ l'auteur et la référence du document. \\ Toute autre reproduction est interdite sauf accord préalable de l'éditeur, en dehors des cas prévus par la législation \\ en vigueur en France.
}

\section{revues.org}

Revues.org est un portail de revues en sciences humaines et sociales développé par le Cléo, Centre pour l'édition électronique ouverte (CNRS, EHESS, UP, UAPV).

\author{
Référence électronique \\ recherchestravaux.revues.org/293 \\ Éditeur : ELLUG \\ http://recherchestravaux.revues.org \\ http://www.revues.org \\ Document accessible en ligne sur : http://recherchestravaux.revues.org/293 \\ Ce document est le fac-similé de l'édition papier. \\ (c) Recherches \& Travaux
}

François Genton, «Le Dictateur de Charlie Chaplin ou le rire de la raison», Recherches \& Travaux [En ligne], 67 | 2005, mis en ligne le 30 septembre 2008, consulté le 16 septembre 2013. URL : http:// 


\section{Le Dictateur de Charlie Chaplin ou le rire de la raison}

Un rire juste

Alors que viennent de prendre fin les cérémonies consacrées au soixantième anniversaire de la libération d'Auschwitz, il peut sembler utile de rappeler tout ce qui a contribué, dans les domaines de la pensée, de la politique et de l'art, à mettre en garde et à mobiliser contre le nazisme, alors que la passivité prévalait, par indifférence ou lâcheté. Le film de Chaplin Le Dictateur (The Great Dictator, 1940)달 ${ }^{\mathrm{I}}$ est dans ce contexte un document de première importance. Cette grande satire soulève deux problèmes liés au défi esthétique et moral que représente le traitement comique d'un régime bien plus monstrueux que Chaplin ne pouvait se l'imaginer. Le premier problème concerne l'analyse politique qui sous-tend le film: n'est-il pas un peu léger de rire d'un régime national-socialiste ramené à la dimension de la dictature exercée par un paranoïaque prénommé «Adenoid " (Adolf + paranoid) sur une masse abêtie? Le second problème, toujours lié au traitement comique du nazisme, porte sur le medium employé, à savoir un film de grande diffusion, destiné à être "consommé» le plus rapidement possible par le public le plus large possible, un produit de cette «culture de masse" qui vise, pour parler avec Hannah Arendt (1989, p. 263 et 27I), à la distraction (entertainment) et non à la cultura animi.

Le Dictateur symbolise, du point de vue de l'histoire politique, la mobilisation d'une fraction croissante de l'opinion publique nord-américaine contre le danger hitlérien. Certes, en I9I8 dans Charlot soldat (Shoulder Arms), Chaplin avait tourné en dérision le militarisme et l'autoritarisme de l'Empire. Ce message de 1918 résonne encore dans le discours final du Dictateur, puisque le petit barbier juif, s'adressant aux foules de l'Osterlich (= l'Autriche)

I. La Paramount s'étant réservé le titre The Dictator, Chaplin a dû ajouter l'adjectif Great. Le titre français est plus conforme à son intention que le titre original. 
envahie, commence ainsi son discours: "Désolé, mais je ne veux pas être empereur, ce n'est pas ma vocation ${ }^{2}$ », rappelant au-delà d'Adenoid Hynkel la figure grotesque de Guillaume II telle que l'avait incarnée en I9I8 Sydney, le demi-frère de Charlie Chaplin. Si dans le film de 1940 le militarisme Hohenzollern se compromet un certain temps avec le nouveau régime, à travers le personnage du général Schultz, c'est cependant bien le nazisme avec ses composantes centrales, l'antisémitisme, l'expansionnisme et le contrôle total de la population, et ses protagonistes d' "un type nouveau» (Hynkel et consorts) qui occupent le devant de la scène, conférant une dimension politique originale au premier long métrage véritablement parlant de Chaplin - et au dernier film avec un avatar de Charlot, à savoir le petit barbier juif.

Dans son autobiographie, Chaplin affirme que c'est le cinéaste d'origine hongroise Alexander Korda qui lui aurait inspiré en 1937 l'idée de départ du scénario de son film: une méprise (mistaken identity) due à la ressemblance du dictateur et du petit barbier juif. Le même Alexander Korda devait produire, sur la même idée de départ, en 1942 To Be or Not to Be d'Ernst Lubitsch, l'autre grande comédie hollywodienne antinazie (Chaplin, p. 386). Hitler avait la moustache de Charlot et, à quatre jours près, la date de naissance de Charlie Chaplin, en avril I889. Le cinéaste a travaillé au scénario du Dictateur dès 1938, avant les accords de Munich et le pogrome du 9 novembre 1938, un événement qui choqua l'opinion publique des États-Unis. Il commença le tournage en janvier 1939 et le termina en juin 1940, au moment de la défaite de la France. Ce n'était pas le premier film antinazi produit par le cinéma nordaméricain. Jusqu'en 1939, l'autorité d'autocensure, le fameux Hays Office, s'efforçait d'émousser les pointes antinazies, comme par exemple dans le film Three Comrades inspiré en 1938 à Frank Borzage par le roman qu'Erich Maria Remarque avait publié en 1936. On considère que la "déclaration de guerre» d'Hollywood à l'Allemagne nazie date de Confessions of a Nazi Spy (1939), un film qui rapporte l'histoire du démantèlement d'un réseau d'espionnage nazi aux États-Unis. Ce film est marqué par une forte implication d'Européens de l'Est et du Centre, du réalisateur Anatole Litvak, originaire d'Ukraine, aux premiers rôles Paul Lukas (Hongrie), Francis (Frantisek) Lederer (Tchécoslovaquie) et Edward G. Robinson (Roumanie).

Le scénario du Dictateur se heurta à deux oppositions: l'une "interne", celle des jeunes assistants de Chaplin, «libéraux» au sens nord-américain du terme, portait sur le comique antihitlérien et surtout sur le sentimentalisme et le pathétique du discours terminal (Robinson, p. 309). L'autre, "externe», provenait des nazis et de leurs amis, mais aussi du gouvernement britannique

2. I'm sorry, but I don't want to be an Emperor - that's not my business. 
qui intervint pour empêcher le film (voir Short) et des «neutralistes» nordaméricains, à commencer par la presse Hearst. La première eut lieu le 3 octobre I940, devant un public choisi comprenant les écrivains Aldous Huxley et John Steinbeck et le réalisateur Lewis Milestone. James Roosevelt, le fils du président des États-Unis, était également présent; lors de la cérémonie d'investiture de Franklin D. Roosevelt, en janvier 1941, Chaplin lut à la radio le discours final du Dictateur. Ce film fut un énorme succès commercial, le plus grand pour Charlie Chaplin, mais dès la sortie on lui reprocha de sous-estimer et de personnaliser la problématique du nazisme (Robinson, p. 32I).

L'idée des sosies permettait à Chaplin de poursuivre sur la voie de l'opposition cinéma muet / cinéma parlant qu'empruntait sa création cinématographique depuis Les Lumières de la ville. À la pantomime du barbier juif s'opposaient les éructations du tyran Hynkel, dont la langue était un savoureux jargon à base d'anglais et d'allemand. Ce dernier rôle donnait au cinéaste l'occasion de replacer des sketches déjà rodés, tel que celui de la danse avec le globe terrestre (Robinson, p. 316). Chaplin a pu se tromper sur plusieurs points, en faisant de Goering (Herring = hareng) un personnage trop grotesque ou en présentant Goebbels (Garbitch = garbage, détritus) seulement comme un froid stratège: cela dit, qui contredirait l'idée que suggèrent leurs noms et, par antiphrase, le jugement d'Adenoid Hynkel (smell good), à savoir qu'il s'agit de personnages peu ragoûtants? Le réalisateur du Dictateur s'est surtout trompé en présentant des camps de concentration et des sbires nazis un tableau presque idyllique (comme il l'a reconnu dans ses mémoires), mais personne en juin 1940 ne savait ce que serait la "solution finale». Il n'en reste pas moins que le cinéaste a su brosser du nazisme le tableau le plus complet. Il fait référence au militarisme allemand, dont la dictature aggrave les traits négatifs, la démesure et le goût pervers des inventions meurtrières: Herring vante même un «formidable» nouveau gaz toxique! Le mérite essentiel du film tient à la place centrale qu'il réserve à l'expansionnisme et à l'antisémitisme du dictateur. Chaplin ne s'est pas embarrassé des diverses explications politiques, géopolitiques, économiques, diplomatiques, philosophiques du nazisme: l'heure des concessions à $\mathrm{M}$. le Chancelier Hitler était révolue.

Quel destin un Hynkel peut-il bien préparer au ghetto? Le discours final n'est qu'une envolée vers l'utopie, le public sait bien qu'Hannah et les siens n'ont pas été sauvés et que le petit barbier juif ne parle que pour exhorter les pays encore libres à intervenir et à mettre fin à la série des succès politiques et militaires de Hitler. S'attaquer aux juifs, c'était s'attaquer à l'humanité entière: Charlot, le vagabond universel, était devenu un juif allemand. Incarnation de la haine raciste et nationaliste, Hynkel est en soi un personnage 
dérisoire, voire risible - ce qui n'empêche pas qu'il soit doté d'une terrifiante capacité de nuire. Il répand autour de soi la mort et viole toute expression authentique de la vie humaine passée, présente et à venir, la sculpture (le «Penseur» de Rodin et la "Vénus de Milo» font le salut nazi!), la danse, la musique, la gastronomie, mais aussi le langage, la science et la technique, l'amour et la famille.

La vision d'en bas propre à Charlot se révèle particulièrement riche et forte. Nous nous contenterons de la rapprocher de deux analyses contemporaines. Le mouvement communiste sous-estimait le racisme et l'antisémitisme du dictateur autrichien en qui il voyait l'agent d'une aile extrême du capitalisme. Thomas Mann pour sa part considérait dès 1939 le dictateur allemand, dans un essai intitulé Frère Hitler (Bruder Hitler), comme une conséquence de la passivité et de l'opportunisme multiséculaires des Allemands d'une part et de l'aventurisme irrationaliste de leurs élites d'autre part. Comme Chaplin, les ennemis politiques du nazisme se réferent à l'universalisme: l'humanité est une, par-delà les différences culturelles, religieuses ou ethniques. Cette idée est au centre même des Lumières, de la représentation du «bon sens, la chose la mieux partagée» (Descartes) au sapere aude de Kant.

C'est lorsque les événements le contraignent à prendre Hitler au sérieux que Chaplin décide de tourner sa comédie sur le dictateur allemand. Si Hynkel prête à rire, c'est parce qu'il pense incarner une forme supérieure d'humanité, alors qu'il n'est qu'un être banal et primitif: tout le comique du film de Chaplin provient du fossé qui sépare les prétentions, le délire du personnage et sa réalité individuelle. Le charisme du tyran ne repose que sur un véritable lavage de cerveau. Vers la fin du Dictateur, Hynkel est arrêté et molesté par les sbires de son régime. Comme il a piètre allure, après être tombé dans l'eau, ces hommes le confondent, malgré son accoutrement tyrolien, avec le petit barbier juif juste évadé du camp de concentration. Hannah Arendt a choqué une partie de l'opinion publique lors du procès Eichmann en parlant de la «banalité du Mal»: appliquée à Eichmann, cette formule signifiait que le Mal ne résidait pas dans le caractère monstrueux du criminel, mais dans sa normalité d'exécutant efficace d'une politique monstrueuse. D'une autre manière, cette formule se vérifierait pour Hitler: s'il ne fut pas un exécutant comme Eichmann, Hitler fut, lui aussi, l'instrument d'une idéologie monstrueuse à laquelle l'évolution politique avait donné les moyens de se convertir en pratique de gouvernement en Allemagne d'abord, dans l'Europe occupée ensuite. En 1946, Peter de Mendelssohn, frappé par la médiocrité des accusés de Nuremberg, réfléchit à ce qui pouvait naguère leur donner une telle apparence de pouvoir et de gloire. Rayonnaient-ils dans la lumière que projetait sur eux le Führer? Non, car Hitler lui-même n'aurait pas plus belle allure s'il 
figurait dans la tribune des accusés (Glaser, p. 353). Le dictateur «nu» ou "dévoilé» de Chaplin ne fait pas illusion: à l'enthousiasme aveugle de la masse manipulée le film substitue le rire de la raison.

\section{Rire éclairé et sombre pensée}

Le XXe siècle a ébranlé la foi dans la possibilité de faire coïncider le progrès des sciences et des techniques avec le progrès de la civilisation. Dès l'époque hitlérienne, de leur exil nord-américain, Horkheimer et Adorno, les philosophes de l'École de Francfort, ont parlé de "dialectique des Lumières». Les Lumières, définies comme une attitude intellectuelle perceptible dès l'Antiquité, se sont imposées aux XVIII et XIX ${ }^{e}$ siècles. En éclairant l'humanité, on voulait la délivrer de la peur et instaurer sa domination sur la nature. Selon Horkheimer et Adorno, c'est le contraire qui s'est produit: «La terre totalement éclairée rayonne sous le signe du malheur triomphant» (Horkheimer / Adorno, p. 9). Comme dans le tableau de Goya, le sommeil de la raison a engendré des monstres. En croyant émanciper l'humanité par la soumission du monde physique, animal et végétal, les Lumières l'ont asservie. Il est de bon ton aujourd'hui de ressasser cette représentation d'un échec des Lumières, particulièrement patent au $\mathrm{XX}^{\mathrm{e}}$ siècle. Des courants en soi aussi différents que le Kulturpessimismus du début du XXe siècle ou la "théorie critique» de Horkheimer et Adorno ont contribué à nourrir une attitude intellectuelle à la fois critique et résignée, puisque tout en "vitupérant l'époque», on juge inepte, voire dangereuse toute tentative politique de changer la situation: n'est-ce pas là la meilleure définition d'une attitude proprement conservatrice?

L'idée d'un échec des Lumières ne date pas de l'École de Francfort: on sait qu'elle est un véritable article de foi pour les milieux conservateurs contemporains de la Révolution française et leur descendance, dans toute l'Europe... et particulièrement en Allemagne. Joachim Fest a publié plusieurs ouvrages sur Hitler et le Troisième Reich, dont le plus récent, Les Derniers Jours d'Hitler (Der Untergang, 2003), a inspiré le film d'Oliver Hirschbiegel La Chute (Der Untergang, 2004). Héritier de la pensée conservatrice allemande, l'ancien responsable des pages culturelles de la Frankfurter Allgemeine Zeitung a déclaré récemment:

L'image que les Lumières se faisaient de l'homme s'est révélée entièrement fausse. Nous nous sommes fait des illusions. Les Lumières ont cru que l'homme était bon et qu'il n'avait qu'à acquérir le savoir approprié et trouver des conditions sociales favorables pour qu'on se dirige vers la paix, le bonheur et la prospérité. Cela n’a rien à voir avec la réalité. Cette belle image a été réfutée par Hitler, par Staline, par Pol Pot, par Idi Amine et d'autres dictateurs. (Die Welt, 2 septembre 2004) 
Cette déclaration est ambiguë: les dictateurs ont-ils «réfuté» les Lumières par l'ampleur des crimes qu'ils ont commis ou parce qu'ils ont fait le malheur des hommes en voulant "des conditions favorables" pour «la paix, le bonheur et la prospérité" ? Qu'elle soit "de droite " ou se dise "critique», la thèse de l'échec des Lumières crée une atmosphère sombre. Pour les auteurs de la Dialectique des Lumières, on ne peut plus maintenir l'illusion d'un art et d'une vie intellectuelle autonomes dans un monde envahi par la technique et le mercantilisme et il est encore moins question de créer un contre-projet enraciné dans la société et susceptible de renverser le cours des choses. Un art et une pensée se voulant critiques n'ont d'autre issue que de se dérober, tout en restant conscients du caractère illusoire de cette dérobade. Cette pose "désillusionnée» marque aussi les nombreuses théories du "décentrement» $\mathrm{du}$ sujet en vogue dans les sciences humaines depuis la Deuxième Guerre mondiale ainsi que dans un art contemporain qui refuse ou pervertit radicalement les formes et les contenus de la "culture de masse»: le sujet n'est plus, au mieux, qu'un effet de la structure ou, au pire, une illusion à "déconstruire».

La critique de la modernité inhérente au cinéma de Charlie Chaplin n’a jamais été jugée recevable par Horkheimer et Adorno. Walter Benjamin, dans son fameux texte sur L'Euvre d'art à l'ère de sa reproductibilité technique (Das Kunstwerk im Zeitalter seiner technischen Reproduzierbarkeit, 1936), avait défini les films de Chaplin comme l'art moderne progressiste par excellence, parce qu'ils permettent à la masse d'accéder à la position de l'amateur d'art éclairé et d'associer le plaisir esthétique à la critique sociale, alors que cette même masse, désorientée, ne peut que rejeter l'art de Picasso:

La reproductibilité technique de l'œuvre d'art transforme la relation de la masse à l'art. Des plus réactionnaires, par exemple face à un Picasso, cette relation devient des plus progressistes, par exemple devant un Chaplin. (Walter Benjamin, p. 159)

Cette idée heurta Adorno. Dès 1936, il écrit ces lignes à Benjamin, à propos des Temps modernes:

Au cinéma le rire du spectateur n'est rien moins que bon et révolutionnaire, mais plein du pire sadisme bourgeois; [...] et, pour relever un autre petit détail, affirmer que le réactionnaire devient avant-gardiste grâce à sa situation de compétence devant un film de Chaplin me semble également relever d'une vision tout à fait romantique, car je ne peux considérer le préféré de Kracauer ${ }^{3}$ (= Chaplin, FG) comme un auteur d'avant-garde, même après Les Temps modernes, et ne pense pas non plus que le moindre des éléments à peu près corrects de ce film puisse être appréhendé consciemment. (Adorno / Benjamin, p. 17I)

3. S. Kracauer (I889-I966) était, entre autres choses, spécialiste du cinéma de la République de Weimar. Juif, il dut s'exiler en I933 en France, en I94I aux États-Unis. 
Quelques années plus tard, dans la Dialectique des Lumières, Adorno ajoute que Chaplin a au moins touché juste par un détail du Dictateur, à savoir la ressemblance du «barbier du ghetto avec le dictateur» (Horkheimer / Adorno, p. 252). En effet, est-il dit, les leaders fascistes ne possèdent pas une individualité d'autant plus marquée que les masses qu'ils dominent en sont dépouillées. Au contraire, ils ne représentent que des personnages de «coiffeurs, cabotins de province ou journalistes à sensation». Dans cette mesure seulement, Chaplin a bien fait de jouer sur l'identité du petit barbier du ghetto et du dictateur: façon inutilement compliquée de dire l'évidence, à savoir que Hitler ne vaut pas plus que le barbier juif... pour insinuer que le barbier juif ne vaut pas davantage que le dictateur. Selon Horkheimer et Adorno, le style même du film de Chaplin montre qu'il y a non seulement identité entre les personnages, mais entre les «messages» :

Les épis de blé flottant au vent dans les champs de la fin du film de Chaplin démentent l'éloge antifasciste de la liberté. Ils ressemblent aux blondes mèches des jeunes Allemandes que l'UFA filme dans leurs camps d'été. En représentant la nature en salutaire opposition à la société, le mécanisme de domination sociale la commercialise, la récupérant pour une société sans salut. Les images qui disent que les arbres sont verts, le ciel bleu et que les nuages passent en font des cryptogrammes de cheminées d'usines et de stations-service. (Horkheimer / Adorno, p. 157)

L'École de Francfort nie la possibilité d'une culture de masse véritablement éclairée: le spectateur des films de Chaplin, loin de soumettre le monde que lui présente le cinéma à une analyse critique, rit du malheur des pauvres et des opprimés, et se contente sans doute du sentimentalisme du happy end pour juger que tout est pour le mieux, confirmant ainsi un univers de cauchemar.

C'est le «bon goût» qui est en cause, un a priori qui reflète l'indignation d'une prétendue avant-garde philosophique allemande face à ce qu'elle ressent comme une intrusion insupportable dans son domaine, celui de la critique de la société moderne. Peu importent les arguments. Lorsqu’il est question du film Les Temps modernes, Adorno reproche à Chaplin de faire rire de son personnage de victime de la société capitaliste technique et industrielle - mais, pour Le Dictateur, l'argument du «sadisme bourgeois» est abandonné, étant donné que c'est précisément cette instrumentalisation de l'être humain qui est au centre de la satire. En 1962, à propos du Dictateur et de la pièce de Brecht La Résistible Ascension d'Arturo Ui (Der aufhaltsame Aufstieg des Arturo Ui), Adorno ne parle plus de sadisme, mais de légèreté:

Non que le respect dû à quelque grandeur historique interdise de rire du peintre en bâtiment (Hitler-FG) ou que le groupe qui s'empara du pouvoir n'ait été une 
bande criminelle. Mais cette affinité élective ne se produit pas hors du territoire, elle est enracinée dans la socialité même. Pour cette raison, rire du fascisme, comme faisait le film de Chaplin, revient immédiatement à ressentir une épouvante extrême. Si l'on escamote cette dimension, si l'on raille les piètres exploiteurs de marchands de primeurs (dans la pièce de Brecht-FG), alors qu'il s'agit de positions-clés, l'attaque ne porte pas. Le Dictateur perd sa force satirique jusqu'à commettre un sacrilège dans la scène où une jeune juive tape avec une poêle sur la tête des SA sans se faire tailler en pièces. (Hembus, p. IIO)

Cette thèse est explicitée en 1967 dans "L'art fait-il rire?» («Ist die Kunst heiter?», Kiedaisch, p. 68 sq.). Bien sûr le fascisme est comique par plus d'un côté, le cabotinage, la bassesse, l'espionnite, etc. Mais rire de ces aspects, c'est revenir en arrière, à une époque où la société recelait des refuges d'où l'on pouvait la moquer, comme aux temps de Schweyk. Ou bien c'est adopter la position du vainqueur, en faisant croire que le fascisme est une cause perdue d'avance, qu'écraseront les puissants bataillons de l'histoire universelle. Or le fascisme, répète Adorno, est né de la société elle-même, de sa profondeur. Adorno condamne le rire de la comédie "populaire», expliquant qu'il est impie de faire rire du fascisme et que les vrais Führer n'étaient pas les clowns, dont les fascistes n'auraient fait qu'imiter après coup (nachträglich) les inepties, ce qui signifie sans doute que Hitler n'était pas une imitation de Chaplin (Kiedaisch, p. 69). On note tout de même une évolution dans les réquisitoires: si en 1944, le film de Chaplin était accusé de tenir au fond le même discours que les films nazis, vingt ans plus tard on lui reproche surtout sa grande légèreté, une attitude moralisatrice dans la lignée de la fameuse formule d'Adorno en I95I: «[...] écrire un poème après Auschwitz est barbare».

S'il est barbare d'écrire un poème après Auschwitz, que dire de ceux qui entreprendraient de rire de cette entreprise d'industrialisation de la mort? Horkheimer et Adorno condamnaient le rire de «l'industrie culturelle» dès 1944:

Le Fun est un bain d'acier. L'industrie du divertissement prescrit inlassablement ce remède. Pour elle, le rire permet de créer une sensation trompeuse de bonheur. Or, les moments heureux se passent du rire, seules les opérettes, puis les films présentent le sexe avec des éclats de rire. Mais un Baudelaire est aussi dénué d'humour qu'un Hölderlin. [...] Rire de quelque chose revient toujours à tourner quelque chose en dérision, et la vie qui selon Bergson transperce le mécanique n'est autre que cette vie barbare qui fait irruption, cette affirmation de soi qui saisit avec audace l'occasion de la joie collective pour célébrer l'abandon de ses scrupules. (Horkheimer/Adorno, p. 153)

Bergson a défini le rire comme l'effet résultant «du mécanique plaqué sur du vivant», le rire réagissant, avec bienveillance, aux obstacles qu’opposent à la souplesse et à la mobilité du vivant la raideur, la corporéité, voire «la 
transfiguration momentanée d'une personne en chose». Le rire, fondé sur une idée positive du "vivant", est une réaction saine. En revanche, les philosophes allemands manifestent un mépris hautain à l'égard de genres populaires auxquels on assigne la seule fonction de contribuer à la déshumanisation de la vie, en quelque sorte une préfiguration de la logique qui a prévalu chez les concepteurs d'Auschwitz. Le rire confirme les pires évolutions. Il est dit dans le même passage que "s'amuser signifie être d'accord [...], ne plus y penser, oublier la souffrance là où on la montre encore».

Avant d'examiner de près les «arguments» d'Adorno et de Horkheimer, il faut rappeler certaines évidences allemandes. Bien avant 1933, la presse d'extrême-droite allemande a attaqué en Chaplin le juif qu'il n'était pas et dès I934 le régime hitlérien a interdit ses films. Adorno et Horkheimer expriment d'ailleurs face au cinéma les préjugés de la petite bourgeoisie allemande "cultivée" (Bildungsbürgertum) et face à Chaplin et aux Lumières en particulier un sentiment hostile qui rejoint celui des milieux nationalistes et antisémites. Les allusions méprisantes au Dictateur dans les écrits des philosophes de l'École de Francfort sont non seulement absurdes, elles reposent aussi sur des souvenirs inexacts. Quand Hannah (Paulette Goddard) frappe les $S A$, ces derniers s'emparent d'abord du barbier juif pour le pendre au réverbère: le général Schultz intervient in extremis, reconnait l'homme qui lui a sauvé la vie et le délivre. Nous sommes dans le registre de la fiction, fiction plutôt "juste» puisque la Wehrmacht (incarnée par le général Schultz) était une institution dont les nazis se méfiaient. Non sans raison, si l'on considère que ce furent des officiers qui conçurent l'attentat du 20 juillet 1944 contre Hitler. À la fin du film, on ne voit pas de champs de blé, mais des vignes: la scène se passe en Osterlich / Autriche. Hannah, une citadine, vient de fuir avec sa famille dans ce paysage certes accueillant, mais qui n'est pas le sien. Dans de nombreux films de Chaplin, la «belle nature» est une représentation ironique, une improbable Arcadie née de l'imagination d'un citadin affamé et malheureux. Nulle ironie toutefois dans la scène finale du Dictateur, car l'on sait bien que la situation de ces juifs réfugiés est désespérée, comme celle de millions de juifs dans l'Europe de 1940. Dans l'Osterlich envahie, Hannah et sa famille sont tombés entre les mains d'une bande hitlérienne. Même ce beau paysage de vignes n'est pas un refuge sûr. Adorno, un musicien averti, a manqué un détail qui aurait pu le servir: la même musique de Richard Wagner (arrangée par Meredith Willson), inspirée de l'ouverture de Lohengrin, accompagne la danse du globe et la scène de la fin. Pourquoi pas? Dans un cas, le pathétique wagnérien marque ironiquement ce que peuvent avoir de comique les ambitions démesurées d'un petit devenu tyran, dans l'autre il renforce l'émotion. C'est le discours qui importe. Le petit 
barbier juif a-t-il tort de dire à Hannah qu'un autre avenir est possible, puis, s'adressant aux hommes libres, de les exhorter à combattre pour la liberté? Se trompe-t-il, avant même de connaître les chambres à gaz et la bombe atomique, lorsqu'il met en garde contre un usage inhumain des nouvelles techniques: "More than machinery, we need humanity"?

Comme l'art "à public restreint", l'art "de masse" véhicule toutes sortes de contenus, et il en va de même pour toutes les formes de comique. L'effort entrepris par Hollywood, à la suite du film de Chaplin, pour mobiliser l'opinion publique nord-américaine contre les puissances de l'Axe a eu un rôle important dans la guerre. Les officiers culturels nord-américains ne s'y trompèrent pas. Ils n'avaient pas besoin de connaittre les thèses de Walter Benjamin sur un art de masse progressiste pour faire diffuser, dès l'été 1945 , les films de Chaplin dans leur zone d'occupation. Dans les documentaires de George Stevens sur la fin de la guerre et le début de l'occupation, les Allemands font la queue devant les cinémas pour voir (ou revoir) The Gold Rush (La Ruée vers l'or). Le Dictateur quant à lui ne fut pas montré avant 1958 dans les cinémas ouest-allemands - et avant 1980 à la télévision est-allemande: 500 notables du théâtre et du cinéma allemands, convoqués par les Américains à Berlin le 9 août 1946, avaient jugé qu'il était prématuré d'infliger aux Allemands une comédie satirique sur un sujet aussi grave. Alfred Andersch se demanda alors si l'on voulait éviter de heurter la sensibilité des ex-nazis (Andersch, p. 8).

\section{Culture «de masse» et génie artistique}

Comme à toute ouvre d'art, on peut appliquer aux films le concept leibnizien de monade tel que le définit Gilles Deleuze:

[...] la monade contient tout, elle exprime le monde entier. Elle exprime l'univers entier. Seulement, attention: elle a une petite région privilégiée qu'elle exprime particulièrement ou clairement. (Cours du 20/05/1987)

Le Dictateur dit le monde de 1940 à partir d'un point de vue universaliste et éclairé. On reste même surpris, plus de six décennies après, de la lucidité de Chaplin. L'accent est mis sur le danger mortel que représentent pour l'humanité, incarnée par les juifs, un régime criminel et son idéologie: la personne du criminel en chef n'a rien d'admirable, ni d'impressionnant, bien au contraire. N'est-ce pas au fond ce que dit aussi le film dramatique d'Oliver Hirschbiegel La Chute (Der Untergang, 2004), qui n'a pas le mérite d'être drôle? Il peut sembler injuste de juger "pervers" (Claude Lanzmann, FranceSoir, 4 janvier 2005) un film ne laissant aucun doute sur la nature criminelle du régime nazi et montrant l'homme Hitler pour ce qu'il était, un paranoïaque malfaisant jusqu'au bout. Ce film dit le monde à partir du point de 
vue raisonnable de l'Allemagne actuelle, confrontée au spectacle repoussant de la décomposition d'une société de fanatiques et de carriéristes sans scrupule dans les derniers jours de la guerre. Est-il révoltant de décrire dans ce chaos quelques personnages positifs (les médecins) ou de suggérer la perspective d'un avenir meilleur pour l'Allemagne à la fin? Wim Wenders a, selon nous, eu tort de reprocher au film une absence de point de vue (Die Zeit 44/2004). Spectacle plutôt bien conçu et juste, le film d'Oliver Hirschbiegel souffre tout au plus de ne rien dire qu'on ne sache déjà. Quant à Hitler, personnage sans grandeur, même négative, il n'y a aucune raison de frapper d'interdit la représentation de sa personne. Les productions de la culture de masse méritent d'être prises au sérieux et l'entertainment n'est pas en soi un crime contre la culture... ni l'élitisme ou l'hermétisme une garantie de sérieux. Le Dictateur est l'exemple même d'une œuvre conçue contre un danger pressant, belle et mobilisatrice, parce qu'elle inclut les moments de l'analyse critique, de la compassion et de l'appel au combat. Par son rôle historique, cette comédie possède un avantage sur les œuvres qui ont raison après coup, surtout quand, contrairement au film de Chaplin, elles arrangent l'Histoire de manière à confirmer les préjugés des auteurs de la Dialectique des Lumières.

Ainsi, le numéro de prisonnier de Roberto Benigni dans La Vie est belle (La Vita è bella, 1998) a beau être le même que celui du petit barbier juif dans son camp de concentration, on peut tout de même regretter l'image trompeuse, si agréable au monde unipolaire d'aujourd'hui, de chars américains libérant un camp de la mort. Comment croire par ailleurs à l'histoire d'un déporté qui parvient à faire retentir la (tout de même banale) «Barcarolle» des Contes d'Hoffmann sur les haut-parleurs du camp? "L'esprit n'est point ému de ce qu'il ne croit pas" a dit Boileau. Le scénario de La Vie est belle s'inspire, entre autres, du roman de Jurek Becker Jacob le menteur (Jakob der Lügner, 1970), porté au cinéma par Frank Beyer (RDA, 1974) et par Peter Kassovitz (USA, 1998). La fable originale de Becker, un vieil homme qui parvient, un peu malgré lui, à préserver le moral d'une petite fille et de tout un ghetto destinés à mourir en leur racontant des histoires, a davantage de force que le film italien. Anémie de l'invention, concessions par anticipation aux attentes "de la masse», telles sont peut-être les caractéristiques du mauvais goût, d'une émotion et d'un rire de mauvais aloi.

Le Dictateur échappe à ces reproches par son inventivité, sa justesse et le courage de son auteur, car Chaplin prenait des risques en entrant aussi résolument dans la sphère politique. Le fun et le kitsch que dénoncent les philosophes de l'École de Francfort existent bien, mais ce n'est pas dans Le Dictateur qu'on les trouvera. Comment ne pas déceler une parenté entre le rire de Chaplin et celui de Molière ou de Voltaire? À défaut d'être un chef-d'œuvre 
absolu, ne serait-ce qu'en raison de la rupture esthétique du discours final, Le Dictateur s'affirme déjà dans une certaine durée comme une ouvre d'art survivant aux périls mêmes qu'elle a dénoncés: il est vrai que, même soixante ans après la capitulation des puissances de l'Axe, il reste beaucoup à faire pour que soient appliqués les principes avancés dans le discours final du barbier juif. Aux côtés de Tartuffe et de Candide, le film de Chaplin parie sur l'efficacité de la satire et sur une humanité capable d'agir en fonction d'une réflexion maîtrisée et universellement communicable: cet appel permanent à la réflexion autonome et à l'action, c'est cela les Lumières.

\section{BIBLIOGRAPHIE}

Adorno Th. W., «Ist die Kunst heiter?», Süddeutsche Zeitung, I5-16 juillet 1967. - «Kulturkritik und Gesellschaft «, dans K.-G. Specht (s.d.), Soziologische Forschungen in unserer Zeit. Ein Sammelwerk. Leopold Wiese zum 75. Geburtstag, Cologne Opladen, 195I, p. 228-24I.

- «Zur Dialektik des Engagements», Die Neue Rundschau, 73 (1962), H. I.

Adorno Th. W. / Benjamin W., Briefwechsel 1928-1940, Francfort, Suhrkamp, 1995. Andersch A., "Chaplin und die Geistesfreiheit", Der Ruf. Unabhängige Blätter der jungen Generation, n ${ }^{\circ} 7$, I5 novembre 1946, p. 8.

Arendt H., "La crise de la culture. Sa portée sociale et politique», traduit par B. Cassin d'après "The Crisis in Culture: Its Social and Its Political Significance», dans La Crise de la culture. Huit exercices de pensée politique, sous la dir. de P. Lévy, Paris, Gallimard (Folio), 1989, p. 253-288 (Between Past and Future: Eight Exercises in Political Thought, 1968).

- Elemente und Ursprünge totaler Herrschaft, Munich, Pieper (SP 645), 1986 ( $\mathrm{I}^{\mathrm{re}}$ éd. 1955, version allemande, augmentée de The Origins of Totalitarianism, 1951).

- Eichmann à Jérusalem. Un rapport sur la banalité du Mal, traduction de Eichmann in Jerusalem. A Report on the Banality of Evil (1963) par A. Guérin, revue par M.-I. Brudny-de Launay, Paris, Gallimard (Folio), I99I

Bazin A., Rohmer É., Charlie Chaplin, Paris, Cerf, 1972.

Benjamin W., Illuminationen. Ausgewählte Schriften, t. I, Francfort, Suhrkamp, 1977 (st 345).

Bergson H., Le Rire. Essai sur la signification du comique, Paris, PUF, 2005 ( $\mathrm{I}^{\mathrm{re}}$ éd. 1900).

Boileau, L'Art poétique (1674), chant III.

Bordat F., Chaplin cinéaste, Paris, Cerf, 1998.

Brecht B., "Der aufhaltsame Aufstieg Arturo Uis», Sinn und Form, 1957, $9^{e}$ année, "Zweites Sonderheft Bertolt Brecht», p. 7-99.

- Arbeitsjournal, Francfort, Suhrkamp, 1973, 2 vol.

- Gesammelte Werke, 20 vol., Francfort, Suhrkamp, 1974. 
Chaplin Ch., My Autobiography, Harmondsworth, Penguin, 1974 (I ${ }^{\text {re }}$ éd. 1964).

Delage Ch., Chaplin. La grande histoire, Paris, Jean-Michel Place, 1998.

Deleuze G., Cours Vincennes-St Denis du 20/05/1987. (http://www.webdeleuze. $\mathrm{com} / \mathrm{php} /$ texte.php?cle=I5I\&groupe=Leibniz\&langue=I)

François É., Schulze Hagen, Deutsche Erinnerungsorte, Munich, C. H. Beck, 200I, 3 vol.

Gáspár S., «Unsympathische Hakenkreuzler. Der Hollywoodfilm und seine Beschäftigung mit dem Nazitum ", Wiener Zeitung 23/2/200I.

Genton F., "Rire de Hitler? À propos du traitement comique du nazisme», Chroniques allemandes, $\mathrm{n}^{\circ}$ 5, 1996, p. 70-I47.

Glaser H., 1945. Ein Lesebuch, Francfort, Fischer, 1995 (Fischer TB I2527).

Hembus J., Charlie Chaplin, Munich, Heyne, I98I.

Horkheimer M., Adorno Th. W., Dialektik der Aufklärung. Philosophische Fragmente, Francfort, Fischer, 1996 ( ( ${ }^{\text {re }}$ éd. 1944).

Kiedaisch P. (textes réunis et présentés par), Lyrik nach Auschwitz? Adorno und die

Dichter, Stuttgart, Reclam (RUB 9363), 1995.

Martin M., Charlie Chaplin, Paris, Seghers, 1966.

Mehran H., Scheide F. (s. d.), The Dictator and the Tramp, Berkeley, University of California Press, 2004.

Mendelssohn P. de, Überlegungen. Vermischte Aufsätze, Hambourg, 1948.

Mitry J., Charlot et la "fabulation chaplinesque», Paris, éditions universitaires, I957.

Robinson D., Chaplin. Sa vie, son art, traduit de l'anglais par Jean-François Chaix, Paris, Ramsay, 1987 (Londres, 1985).

Sadoul G., Vie de Charlot, Paris, Lherminier, 1978 ( $\mathrm{I}^{\mathrm{re}}$ éd. 1952).

Short K. R. M., "Chaplin's The Great Dictator and British Censorship, 1939", Historical Journal of Film, Radio and Television V/I, mars I985, p. 85-90.

\section{FILMOGRAPHIE}

Le Dictateur, 2 DVD, mk2, ASIN Boooo6IWVL. Cette édition contient, entre autres "bonus ", The Tramp and the Dictator (2002), le documentaire de K. Brownlow et M. Kloft.

Le site du projet du Fritz-Bauer-Institut « Cinematographie des Holocaust » répertorie I530 films sur le nazisme: http://www.cine-holocaust.de/index.html 